\title{
A SVD-Based Method to Assess the Uniqueness and Accuracy of SPECT Geometrical Calibration
}

\author{
Tianyu Ma, Member, IEEE, Rutao Yao*, Member, IEEE, Yiping Shao, Member, IEEE, and Rong Zhou
}

\begin{abstract}
Geometrical calibration is critical to obtaining high resolution and artifact-free reconstructed image for SPECT and CT systems. Most published calibration methods use analytical approach to determine the uniqueness condition for a specific calibration problem, and the calibration accuracy is often evaluated through empirical studies. In this work, we present a general method to assess the characteristics of both the uniqueness and the quantitative accuracy of the calibration. The method uses a singular value decomposition (SVD) based approach to analyze the Jacobian matrix from a least-square cost function for the calibration. With this method, the uniqueness of the calibration can be identified by assessing the nonsingularity of the Jacobian matrix, and the estimation accuracy of the calibration parameters can be quantified by analyzing the SVD components. A direct application of this method is that the efficacy of a calibration configuration can be quantitatively evaluated by choosing a figure-of-merit, e.g., the minimum required number of projection samplings to achieve desired calibration accuracy. The proposed method was validated with a slit-slat SPECT system through numerical simulation studies and experimental measurements with point sources and an ultra-micro hot-rod phantom. The predicted calibration accuracy from the numerical studies was confirmed by the experimental point source calibrations at $\sim 0.1 \mathrm{~mm}$ for both the center of rotation (COR) estimation of a rotation stage and the slit aperture position (SAP) estimation of a slit-slat collimator by an optimized system calibration protocol. The reconstructed images of a hot rod phantom showed satisfactory spatial resolution with a proper calibration and showed visible resolution degradation with artificially introduced $0.3 \mathrm{~mm}$ COR estimation error. The proposed method can be applied to other SPECT and CT imaging systems to analyze calibration method assessment and calibration protocol optimization.
\end{abstract}

Index Terms-Geometrical calibration, Jacobian matrix, single photon emission computed tomography (SPECT), singular value decomposition (SVD), slit-slat collimator.

\section{INTRODUCTION}

$\mathbf{M}$ ODERN micro-SPECT systems with submillimeter spatial resolution have become essential components in high-resolution small animal imaging [1]-[6]. To achieve

Manuscript received November 24, 2008; revised March 12, 2009. First published June 23, 2009; current version published November 25, 2009. This work was supported by the Roswell Park Alliance Foundation under Grant 62-2426-01. Asterisk indicates corresponding author

T. Ma was with the Department of Nuclear Medicine, State University of New York, Buffalo, NY 14214 USA. He is now with Department of Engineering Physics, Tsinghua University, Beijing 100084, China (e-mail: maty@ tsinghua. edu.cn).

*R. Yao is with Department of Nuclear Medicine, State University of New York, Buffalo, NY 14214 USA (e-mail: rutaoyao@buffalo.edu).

Y. Shao was with Department of Nuclear Medicine, State University of New York, Buffalo, NY 14214 USA. He is now with the Department of Imaging Physics, University of Texas M.D. Anderson Cancer Center, Houston, TX 77030 USA (e-mail: yiping.shao@di.mdacc.tmc.edu).

R. Zhou is with Department of Engineering Physics, Tsinghua University, Beijing 100084, China.

Color versions of one or more of the figures in this paper are available online at http://ieeexplore.ieee.org.

Digital Object Identifier 10.1109/TMI.2009.2025696 high-resolution and artifact-free SPECT images, the geometrical parameters must be known very accurately through system geometrical calibration procedures [7]-[10].

Many geometrical calibration methods have been proposed for fan beam and cone beam SPECT systems in the last 20 years [7]-[11], and more recently, for micro-SPECT systems that use pinhole collimation, which is a special case of cone beam geometry [12]-[17]. Noo et al. [11] described the three basic steps of calibration procedure as follows.

Step 1) Place one or multiple point objects in the field of view and measure the tomographic projections of the objects. The relative locations of multiple objects can be measured and used as a priori knowledge.

Step 2) Establish a geometrical model that can analytically calculate the objects' projections with the to-be-calibrated geometric parameters.

Step 3) Find the optimal solution (such as the least square solution or maximum likelihood solution) for the geometrical parameters by fitting the measured with the predicted projection data.

Usually, most of the proposed calibration methods follow the procedure of the above three steps and apply it to a specific system. Many studies focus on optimizing only one of the above three steps to obtain a unique and accurate solution for the calibration problem. For example, the challenges for step 1 are that some geometrical parameters are highly correlated and difficult to solve for a unique solution with a single point object setup, so Bequé et al. [12] studied the uniqueness condition analytically for a single-pinhole SPECT camera with a circular detector orbit, and derived the numbers of point objects and the specific a priori knowledge needed to provide adequate additional information for detaching the correlation. As for step 2, Wang and Tsui [13] introduced a homogeneous coordinates framework that facilitated the geometrical modeling of nonstandard collimator and acquisition geometries. Based on this framework, the uniqueness condition can be analyzed easily. Defrise et al. [16] proposed a method to consider the perturbation of camera's motion in the geometrical model. When the perturbation was small and with certain hypotheses, the model was linearized and a truncated SVD approach was used to calculate the Moore-Penrose solution of the corresponding minimization problem. Since the typical implementation of step 3 was to seek the direct parameter estimation through a nonlinear fitting procedure [8], [10], [14], [17], this approach may face numerical challenges such as local minima, initial value dependence and ill-conditioness. To avoid these problems, Noo et al. [11] first fitted the raw projection data with an ellipse, then calculated the geometrical parameters from the parameters of the ellipse. Von 
Smekal et al. [18] proposed to use the first three components of the Fourier series of the projection data for the nonlinear fitting.

To design an efficient calibration protocol, the uniqueness condition of a calibration method has been investigated with an analytic model [12], [17]. However, calibration may be numerically inaccurate, even when the analytically derived uniqueness condition is satisfied. For example, during the development of a calibration method for a slit-slat SPECT system (Section III-A), we observed that the estimations of certain parameters were always accurate, whereas the solutions of some other parameters were unstable between experiments. In general, the accuracy is also strongly dependent on the configuration of the calibration experiments, e.g., how many point sources are used, where the point sources are located, how many projections are sampled for each source, and what kind of a priori knowledge is applied.

The main purpose of this paper is to develop a general method to numerically describe the characteristics of both uniqueness and numerical accuracy characteristics of a given geometrical calibration. With this method, we can elucidate the experimental observation, predict achievable calibration accuracy for a given experimental design, and compare different calibration procedures to find the optimal one.

This paper is organized as follows. Section II describes the theory of this work. It consists of two parts: 1) derivation of the relationship between the errors in the estimated calibration parameters and the noise in the measured data and 2) analysis of the Jacobian matrix with singular value decomposition (SVD) technique to characterize the uniqueness and accuracy of calibration. Section III introduces the test case for the proposed method. It includes the description of a slit-slat SPECT system, the system's geometrical projection model and the analytical derivation of calibration uniqueness condition. Section IV applies the proposed method to the slit-slat system, and reports the results of uniqueness and accuracy analysis and calibration optimization. Section $\mathrm{V}$ describes the experimental validation of the proposed method, followed by Discussion and the Conclusion.

\section{THEORY}

\section{A. Least Square Cost Function and Jacobian Matrix}

For a typical calibration method as described in the introduction, the goal is to find the calibration parameter set $\Gamma^{\dagger}$ that satisfies

$$
\Gamma^{\dagger}=\arg \min _{\Gamma} F(\Gamma)
$$

where the vector $\Gamma$ is the ensemble of parameters to be calibrated, and $F(\boldsymbol{\Gamma})$ is the cost function that measures the difference between the measured and predicted projection centroids of one or multiple point sources.

In this study we use a common least square cost function

$$
\begin{aligned}
& F(\boldsymbol{\Gamma})=\frac{1}{2} \sum_{i=1}^{m}\left(p_{i}(\boldsymbol{\Gamma})-c_{i}\right)^{2}=\frac{1}{2} \sum_{i=1}^{m} f_{i}(\boldsymbol{\Gamma})^{2} \\
& f_{i}(\boldsymbol{\Gamma})=p_{i}(\boldsymbol{\Gamma})-c_{i}
\end{aligned}
$$

where $i$ is the projection index, $m$ is the total number of projections, $p_{i}$ is the predicted projection location of an ideal point source, and $c_{i}$ is the centroid for the measured projection.
In an ideal noise-free situation, the true value of $\Gamma$, denoted as $\Gamma^{*}$, and the noise free observation $c_{i}^{*}$ have the following relationship:

$$
c_{i}^{*}=p_{i}\left(\Gamma^{*}\right) .
$$

In the presence of noise

$$
c_{i}=c_{i}^{*}+e_{i}=p_{i}\left(\boldsymbol{\Gamma}^{*}\right)+e_{i} .
$$

By assuming that measurement of projections are independent to each other and there exists no systematic error in (5), we use a Gaussian function with zero mean and variance $\sigma_{i}^{2}$ to model $e_{i}$. This model is similar to that in Bequé et al.'s work [12]. Let $\Gamma^{\dagger}$ be the realistic parameter set estimated from noisy data, and $\Delta$ be the estimation error, then

$$
\Gamma^{\dagger}=\Gamma^{*}+\Delta \text {. }
$$

The first-order Taylor expansion of $f_{i}\left(\boldsymbol{\Gamma}^{*}\right)$ gives

$$
f_{i}\left(\boldsymbol{\Gamma}^{*}\right) \approx f_{i}\left(\boldsymbol{\Gamma}^{\dagger}\right)+\left.\sum_{j=1}^{n} \frac{\partial f_{i}(\boldsymbol{\Gamma})}{\partial \Gamma_{j}}\right|_{\Gamma=\Gamma^{\dagger}}\left(\Gamma_{j}^{*}-\Gamma_{j}^{\dagger}\right)
$$

where $j=1, \ldots, n$ is the parameter index, $n$ is the total number of parameters, and $\Gamma_{j}$ denotes the $j$ th element of $\boldsymbol{\Gamma}$. Since $f_{i}\left(\boldsymbol{\Gamma}^{*}\right)=p_{i}\left(\boldsymbol{\Gamma}^{*}\right)-c_{i}=-e_{i}$, (7) can be rewritten as

$$
\left.f_{i}\left(\boldsymbol{\Gamma}^{\dagger}\right) \approx \sum_{j=1}^{n} \frac{\partial f_{i}(\boldsymbol{\Gamma})}{\partial \Gamma_{j}}\right|_{\boldsymbol{\Gamma}=\Gamma^{\dagger}} \cdot \Delta_{j}-e_{i} .
$$

With (2) and (8), the minimization equations of $F(\boldsymbol{\Gamma})$

$$
\left.\frac{\partial F(\boldsymbol{\Gamma})}{\partial \Gamma_{j}}\right|_{\Gamma=\Gamma^{\dagger}}=0, \quad j=1,2, \ldots, n
$$

are approximated by

$$
\begin{array}{r}
\left.\sum_{i=1}^{m}\left(\left.\sum_{r=1}^{n} \frac{\partial f_{i}(\boldsymbol{\Gamma})}{\partial \Gamma_{r}}\right|_{\Gamma=\Gamma^{\dagger}} \cdot \Delta-e_{i}\right) \frac{\partial f_{i}(\boldsymbol{\Gamma})}{\partial \Gamma_{j}}\right|_{\Gamma=\Gamma^{\dagger}}=0 \\
j=1,2, \ldots, n .
\end{array}
$$

Rearrangement of (10) yields the normal equations

$$
J^{\mathrm{T}} J \boldsymbol{\Delta}=J^{\mathrm{T}} \mathbf{e}
$$

where $\mathbf{e}$ is the ensemble of $e_{i}$, and $J$ is known as the Jacobian matrix, which contains the first partial derivatives of $f_{i}(\Gamma)$

$$
J_{i j}=\left.\frac{\partial f_{i}(\boldsymbol{\Gamma})}{\partial \Gamma_{j}}\right|_{\Gamma=\Gamma^{\dagger}} .
$$

Equation (11) reveals the propagation of error from the measurement noise $\mathbf{e}$ to the parameter estimation $\Delta . J$ plays a key role in the calibration outcome.

1) In the noise-free case, the normal equations are

$$
J^{\mathrm{T}} J \boldsymbol{\Delta}=0 .
$$

So when J is nonsingular, (13) only has zero solution $\Delta=$ 0 , which means the estimated solution $\Gamma^{\dagger}$ is exactly $\Gamma^{*}$. If 
$J$ is a singular matrix, (13) has multiple nonzero solutions and the calibration problem is considered to be nonunique.

2) In the presence of noise, and when $J$ is ill-conditioned, according to (11), a small amount of measurement noise $\mathbf{e}$ will lead to a large error on parameter estimation by a factor of $\left(J^{\mathrm{T}} J\right)^{-1} J^{\mathrm{T}}$, and therefore an inaccurate calibration.

\section{B. Calibration Analysis With SVD}

We use SVD to characterize the uniqueness and accuracy of the calibration problem. With SVD, the $m \times n$ Jacobian matrix $J$ can be decomposed as

$$
\begin{aligned}
& J=U S V^{\mathrm{T}}=\left[\mathbf{u}_{1}, \mathbf{u}_{2}, \ldots, \mathbf{u}_{m}\right] \\
& \times\left[\begin{array}{cccc}
s_{1} & & & \\
& s_{2} & & \\
& & \ddots & \\
& & & s_{n}
\end{array}\right] \times\left[\mathbf{v}_{1}, \mathbf{v}_{2}, \ldots, \mathbf{v}_{n}\right]^{\mathrm{T}} \\
& =\sum_{j=1}^{n} s_{j} \mathbf{u}_{j} \mathbf{v}_{j}^{\mathrm{T}}
\end{aligned}
$$

where $U$ is a $m \times m$ unitary matrix, $S$ is a $m \times n$ diagonal matrix, and $V$ is a $n \times n$ unitary matrix. $\left\{\mathbf{u}_{i}\right\}, i=1, \ldots, m$ and $\left\{\mathbf{v}_{j}\right\}, j=1, \ldots, n$ are called the left-singular and rightsingular vectors, respectively, and $s_{1} \geqslant s_{2} \geqslant \cdots \geqslant s_{n}$ are the singular values. With (14), one can derive the solution of (11) as

$$
\boldsymbol{\Delta}=\frac{1}{s_{1}} \mathbf{u}_{1}^{\mathrm{T}} \mathbf{e v}_{1}+\cdots+\frac{1}{s_{r}} \mathbf{u}_{r}^{\mathrm{T}} \mathbf{e v}_{r}+c_{1} \mathbf{v}_{r+1}+\cdots+c_{n-r} \mathbf{v}_{n}
$$

where $s_{r}$ is the smallest nonzero singular value $(r \leqslant n)$, and $c_{1}, \ldots, c_{n-r}$ can take any values. So with (15), the uniqueness condition of calibration can be simply expressed as $r=n$, or $J$ is nonsingular. When $J$ is singular, those parameters that have nonzero components in $\left\{\mathbf{v}_{r+1}, \ldots, \mathbf{v}_{n}\right\}$ can not be uniquely determined from calibration.

According to (15), when $r=n$, the impact of error propagation is mainly decided by reciprocals of the singular values. In this case, the covariance matrix $\operatorname{cov}\left(\boldsymbol{\Gamma}^{\dagger}\right)=\operatorname{cov}(\boldsymbol{\Delta})$ is used to evaluate the variation of calibration. The variance and covariance of the parameters are the diagonal and off-diagonal elements in $\operatorname{cov}(\boldsymbol{\Delta})$ respectively, which are expressed as

$$
\begin{aligned}
\operatorname{cov}(\Delta)_{k l} \\
\triangleq E\left(\Delta_{k} \Delta_{l}\right) \\
=\mathrm{E}\left\{\left[\left(J^{\mathrm{T}} J\right)^{-1} J^{\mathrm{T}} \mathbf{e}\right]_{k}\left[\left(J^{\mathrm{T}} J\right)^{-1} J^{\mathrm{T}} \mathbf{e}\right]_{l}\right\} \\
=\mathrm{E}\left\{\sum_{i=1}^{m}\left[\left(J^{\mathrm{T}} J\right)^{-1} J^{\mathrm{T}}\right]_{k i}\left[\left(J^{\mathrm{T}} J\right)^{-1} J^{\mathrm{T}}\right]_{l i} e_{i}^{2}\right\} \\
=\left[\left(J^{\mathrm{T}} J\right)^{-1} J^{\mathrm{T}} \operatorname{diag}\left\{\sigma_{1}^{2}, \sigma_{2}^{2}, \ldots, \sigma_{m}^{2}\right\} J\left(J^{\mathrm{T}} J\right)^{-1}\right]_{k l} .
\end{aligned}
$$

If all the measurements have the same variance $\sigma_{c}^{2}=\sigma_{1}^{2}=$ $\sigma_{2}^{2}=\cdots=\sigma_{m}^{2}$, with the SVD expression of $J$ in (14), one can

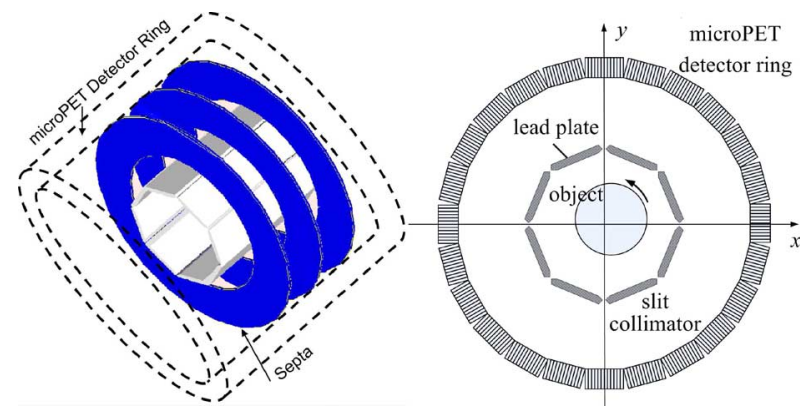

Fig. 1. These two diagrams illustrate the concept of placing a collimator insert inside a PET detector ring for SPECT imaging. The 3-D view on the left shows the relative positioning of the slit-plates and the annular septa (only three septa are shown for clarity). The 2-D transverse view on the right shows the collimator and the detector ring in one plane (septa are not shown).

derive a straightforward relationship between $\operatorname{cov}\left(\Gamma^{\dagger}\right)$ and the singular values and singular vectors of $J$ as the following:

$$
\begin{aligned}
\operatorname{cov}\left(\Gamma^{\dagger}\right) & =\sigma_{c}^{2}\left(J^{\mathrm{T}} J\right)^{-1} \\
& =\sigma_{c}^{2} \sum_{j=1}^{n} \frac{1}{s_{j}^{2}} \mathbf{v}_{j} \mathbf{v}_{j}^{\mathrm{T}} .
\end{aligned}
$$

If different measurement data have different variances, a weight $w_{i}=1 / \sigma_{i}$ can be applied in the original definition of least square cost function in (2), and similar expression of the covariance matrix can still be derived. So, we simply discuss the equal-variance case in this paper. According to [7] and [12], a predefined value of $\sigma_{c}=0.2 \mathrm{~mm}$ is used in the following sections.

From (17), the standard deviation (STD) of the $j$ th parameter $\sigma_{\Gamma_{j}}$ is given by the square root of the $j$ th diagonal element of $\operatorname{cov}(\boldsymbol{\Gamma})$

$$
\sigma_{\Gamma_{j}}=\sigma_{c} \sqrt{\sum_{r=1}^{n}\left(\frac{V_{j r}}{s_{r}}\right)^{2}} .
$$

Therefore, $\sigma_{\Gamma_{j}}$ is determined by the singular values of $J$ and the $j$ th row vector of $V$. With $\sum_{r=1}^{n} V_{j r}^{2}=1$ because $V$ is unitary, if all the $s_{r}$ are large enough, or for some of the parameters the small singular values $s_{r}$ are combined with small $V_{j r}$, one can expect an accurate estimate of those parameters. Otherwise the corresponding parameters may be calibrated with low accuracy.

\section{SLIT-Slat SPECT System AND ANALYTICAL ANAlysis}

\section{A. Slit-Slat SPECT}

The calibration work was initially motivated by the development of a slit-slat animal SPECT system based on a microPET scanner [19], [20]. So the calibration method developed was naturally tested on this system. Fig. 1 illustrates the setup of a collimator insert within the PET detector ring. The PET scanner consists of 4 detector rings and each detector ring has 24 detector blocks. Each detector block consists of a $12 \times 12$ array of 


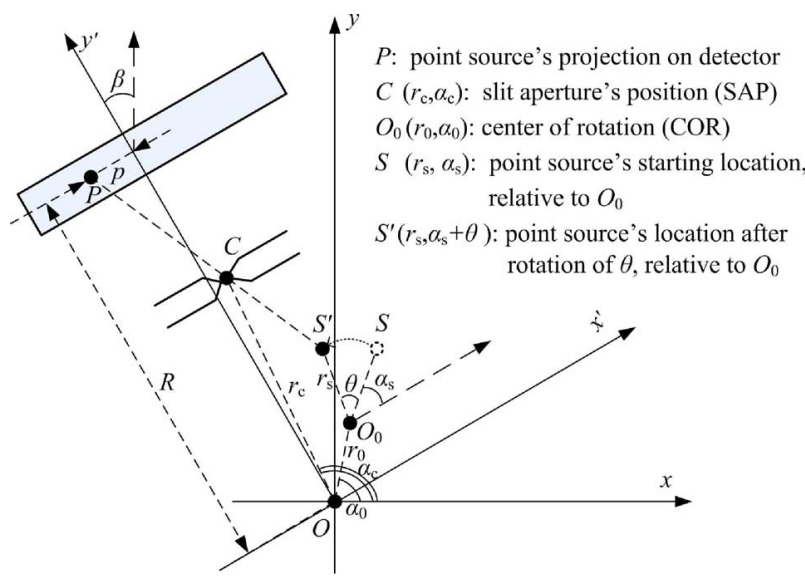

Fig. 2. Geometrical definitions of the coordinate system and the symbols.

Lutetium Oxyorthosilicate (LSO) crystals. The collimator consists of 8 slits and 70 septa. In this study, several assumptions are made on the geometry of the system.

1) The detector blocks in each ring are assumed to have ideal polygon-shape geometry, and the center of the polygon is aligned with the origin of the system. The surface of each block is assumed to be parallel to the axial axis of the PET system. The LSO crystal arrays are assumed to have perfect alignment. As the location of each event is determined from crystal position map rather than using Anger logic and according to the above assumptions, impact of the electronic shift is neglected in this paper.

2) Each slit is parallel to the axial axis of the PET system. The plane of the object's circular orbit and the slats (septa) are assumed to be perpendicular to the axial axis. Although the slits and the slats in the real collimator may be tilted from their ideal positions, they can be calibrated separately. In this work, we focus on the calibration issues in one transverse plane.

\section{B. Geometrical Projection Model}

The definitions of the in-plane coordinate system $(x, y)$ and important geometrical parameters are described in Fig. 2. For illustration, only one slit and one rotating point source are shown. The point source is rotated by an angle $\theta$ from its starting location $S$ to the present location $S^{\prime}$, and is projected through the slit aperture $C$ to the detector. $P$ is the centroid of the projection. The origin of the coordinate system and the COR are denoted by $O$ and $O_{0}$, respectively.

Given that PET scanner's geometry is standard and precisely known, each detector block can be identified only by it's relative orientation angle $\beta$, which is defined as the angle between the $y$ axis and the detector's normal vector. By rotating the coordinate system by $\beta$ counterclockwise, which is shown as $\left(x^{\prime}, y^{\prime}\right)$ in Fig. 2, The cartesian coordinates of $O_{0}, S^{\prime}, C$ and $P$ in the new coordinate system $\left(x^{\prime}, y^{\prime}\right)$ are expressed as

$$
\begin{aligned}
O_{0}= & {\left[r_{0} \cos \left(\alpha_{0}-\beta\right), r_{0} \sin \left(\alpha_{0}-\beta\right)\right] } \\
S^{\prime}= & {\left[r_{0} \cos \left(\alpha_{0}-\beta\right)+r_{s} \cos \left(\alpha_{s}+\theta\right)\right.} \\
& \left.r_{0} \sin \left(\alpha_{0}-\beta\right)+r_{s} \sin \left(\alpha_{s}+\theta\right)\right] \\
C= & {\left[r_{c} \cos \left(\alpha_{c}-\beta\right), r_{c} \sin \left(\alpha_{c}-\beta\right)\right] }
\end{aligned}
$$

and

$$
P=[p, R]
$$

where $p$ is the distance between the point source's projection point $P$ and the $y^{\prime}$ axis, and $R$ is the distance from the origin of the coordinate system to the projection point, which is equal to the sum of the radius of PET's detector ring $(73.6 \mathrm{~mm})$ and the depth-of-interaction (DOI) of a gamma photon detected inside the detector crystal. We simulated a uniform cylindrical ${ }^{99 m} \mathrm{Tc}$ source which filled the entire FOV of this system, and calculated an averaged value of DOI $=1.489 \mathrm{~mm}$ over all the acquired single events within SPECT energy window (120-160 $\mathrm{keV})$. This value is used throughout this paper.

The fact that $S^{\prime}, C$ and $P$ fall on one straight line leads to

$$
\begin{aligned}
& \frac{r_{c} \sin \left(\alpha_{c}-\beta\right)-R}{r_{c} \cos \left(\alpha_{c}-\beta\right)-p} \\
& \quad=\frac{r_{0} \sin \left(\alpha_{0}-\beta\right)+r_{s} \sin \left(\alpha_{s}+\theta\right)-r_{c} \sin \left(\alpha_{c}-\beta\right)}{r_{0} \cos \left(\alpha_{0}-\beta\right)+r_{s} \cos \left(\alpha_{s}+\theta\right)-r_{c} \cos \left(\alpha_{c}-\beta\right)}
\end{aligned}
$$

which yields

$$
\begin{aligned}
p= & r_{c} \cos \left(\alpha_{c}-\beta\right) \\
& -\frac{r_{0} \cos \left(\alpha_{0}-\beta\right)+r_{s} \cos \left(\alpha_{s}+\theta\right)-r_{c} \cos \left(\alpha_{c}-\beta\right)}{r_{0} \sin \left(\alpha_{0}-\beta\right)+r_{s} \sin \left(\alpha_{s}+\theta\right)-r_{c} \sin \left(\alpha_{c}-\beta\right)} \\
& \times\left(r_{c} \sin \left(\alpha_{c}-\beta\right)-R\right) .
\end{aligned}
$$

For a SPECT system with $k$ slits and when $L$ point sources are used in the calibration, the geometrical parameter set $\boldsymbol{\Gamma}$ to be calibrated include

1) $\operatorname{SAP}\left(r_{c k}, \alpha_{c k}\right)$, where $k=1, \ldots, K$;

2) $\operatorname{COR}\left(r_{0 l}, \alpha_{0 l}\right)$, the radius of rotation (ROR) $r_{s l}$, and the starting angular position of the point source $\alpha_{s l}$, where $l=1, \ldots, L$.

In the real experimental setup, only one point source was used which was attached to a rotation stage, and by mounting the stage on the animal bed it can be moved in the axial direction. Projections for "multiple" point sources were acquired by moving the point source to different locations. So each point source may have its own orbit and different $\operatorname{COR}\left(r_{0 l}, \alpha_{0 l}\right)$.

One should note that derivation of (20) is based on rotating the object. If the detector is rotated and the object remains stationary, the projection model is different than (20) which may lead to different uniqueness and accuracy assessment conclusions.

\section{Analytical Analysis of Uniqueness Condition}

According to [12], the uniqueness condition is expressed as: if for any rotation angle $\theta$ and two parameter sets $\Gamma, \widetilde{\Gamma}$, the two sets have to be identical to satisfy the equation

$$
p_{\theta}(\boldsymbol{\Gamma})=p_{\theta}(\widetilde{\boldsymbol{\Gamma}}), \forall \theta
$$

then the calibration problem has a unique solution.

With one point source and one slit, the two parameter sets are $\boldsymbol{\Gamma}=\left(r_{c}, \alpha_{c}, r_{s}, \alpha_{s}, r_{0}, \alpha_{0}\right)$ and $\widetilde{\boldsymbol{\Gamma}}=\left(\widetilde{r}_{c}, \widetilde{\alpha}_{c}, \widetilde{r}_{s}, \widetilde{\alpha}_{s}, \widetilde{r}_{0}, \widetilde{\alpha}_{0}\right)$. 
When the system has only one slit, without losing generality, $\beta$ can be assumed to be 0 , and thus (20) becomes

$$
\begin{aligned}
p= & r_{c} \cos \alpha_{c} \\
& -\frac{r_{0} \cos \alpha_{0}+r_{s} \cos \left(\alpha_{s}+\theta\right)-r_{c} \cos \alpha_{c}}{r_{0} \sin \alpha_{0}+r_{s} \sin \left(\alpha_{s}+\theta\right)-r_{c} \sin \alpha_{c}} \\
& \times\left(r_{c} \sin \alpha_{c}-R\right) .
\end{aligned}
$$

Through a similar derivation as in [12], one can derive the following relationships from (21) and (22) as demonstrated in the Appendix

$$
\begin{aligned}
r_{c} & =\widetilde{r}_{c} \\
\alpha_{c} & =\widetilde{\alpha}_{c} \\
\alpha_{s} & =\widetilde{\alpha}_{s} \\
\widetilde{r}_{s} & =k r_{s} \\
\widetilde{r}_{0} \cos \widetilde{\alpha}_{0}-r_{c} \cos \alpha_{c} & =k\left(r_{0} \cos \alpha_{0}-r_{c} \cos \alpha_{c}\right) \\
\widetilde{r}_{0} \sin \widetilde{\alpha}_{0}-r_{c} \sin \alpha_{c} & =k\left(r_{0} \sin \alpha_{0}-r_{c} \sin \alpha_{c}\right) .
\end{aligned}
$$

Equations (26)-(28) reveal that $r_{s}, r_{0}$ and $\alpha_{0}$ can not be determined uniquely unless there exists an additional condition that provides a constraint of $k \equiv 1$. Therefore one source is not enough to calibrate a single slit.

If the system has multiple slits, one can choose any two of the slits, which are denoted as $\left(r_{c 1}, \alpha_{c 1}\right)$ and $\left(r_{c 2}, \alpha_{c 2}\right)$, then the corresponding parameter sets are $\Gamma=\left(r_{c 1}, \alpha_{c 1}, r_{c 2}, \alpha_{c 2}, r_{s}, \alpha_{s}, r_{0}, \alpha_{0}\right)$ and $\widetilde{\Gamma}=\left(r_{c 1}, \alpha_{c 1}, r_{c 2}, \alpha_{c 2}, \widetilde{r}_{s}, \alpha_{s}, \widetilde{r}_{0}, \widetilde{\alpha}_{0}\right)$. Similar to (28), one can derive that

$$
\begin{aligned}
& \widetilde{r}_{0} \cos \left(\widetilde{\alpha}_{0}-\beta_{1}\right)-r_{c 1} \cos \left(\alpha_{c 1}-\beta_{1}\right) \\
& =k_{1}\left(r_{0} \cos \left(\alpha_{0}-\beta_{1}\right)-r_{c 1} \cos \left(\alpha_{c 1}-\beta_{1}\right)\right) \\
& \widetilde{r}_{0} \sin \left(\widetilde{\alpha}_{0}-\beta_{1}\right)-r_{c 1} \sin \left(\alpha_{c 1}-\beta_{1}\right) \\
& =k_{1}\left(r_{0} \sin \left(\alpha_{0}-\beta_{1}\right)-r_{c 1} \sin \left(\alpha_{c 1}-\beta_{1}\right)\right) \\
& \widetilde{r}_{0} \cos \left(\widetilde{\alpha}_{0}-\beta_{2}\right)-r_{c 2} \cos \left(\alpha_{c 2}-\beta_{2}\right) \\
& =k_{2}\left(r_{0} \cos \left(\alpha_{0}-\beta_{2}\right)-r_{c 2} \cos \left(\alpha_{c 2}-\beta_{2}\right)\right) \\
& \widetilde{r}_{0} \sin \left(\widetilde{\alpha}_{0}-\beta_{2}\right)-r_{c 2} \sin \left(\alpha_{c 2}-\beta_{2}\right) \\
& =k_{2}\left(r_{0} \sin \left(\alpha_{0}-\beta_{2}\right)-r_{c 2} \sin \left(\alpha_{c 2}-\beta_{2}\right)\right)
\end{aligned}
$$

where $\beta_{1}$ and $\beta_{2}$ are orientation angles of the corresponding detector panels facing the two slits. Apparently $k_{1}=k_{2}$ since only one source is used. Equations (29) and (30) can be rewritten as

$$
\begin{aligned}
& \widetilde{r}_{0} \cos \left(\widetilde{\alpha}_{0}-\beta_{1}\right) \\
& \quad=k_{1} r_{0} \cos \left(\alpha_{0}-\beta_{1}\right)-\left(k_{1}-1\right) r_{c 1} \cos \left(\alpha_{c 1}-\beta_{1}\right) \\
& \widetilde{r}_{0} \sin \left(\widetilde{\alpha}_{0}-\beta_{1}\right) \\
& \quad=k_{1} r_{0} \sin \left(\alpha_{0}-\beta_{1}\right)-\left(k_{1}-1\right) r_{c 1} \sin \left(\alpha_{c 1}-\beta_{1}\right)
\end{aligned}
$$

Equation (33) $\times \sin \beta_{1}+(34) \times \cos \beta_{1}$ gives

$$
\widetilde{r}_{0} \sin \widetilde{\alpha}_{0}=k r_{0} \sin \alpha_{0}-(k-1) r_{c 1} \sin \alpha_{c 1}
$$

and (33) $\times \cos \beta_{1}+(34) \times \sin \beta_{1}$ gives

$$
\widetilde{r}_{0} \cos \widetilde{\alpha}_{0}=k r_{0} \cos \alpha_{0}-(k-1) r_{c 1} \cos \alpha_{c 1} .
$$

Similarly, from (31) and (32) one has

$$
\widetilde{r}_{0} \sin \widetilde{\alpha}_{0}=k r_{0} \sin \alpha_{0}-(k-1) r_{c 2} \sin \alpha_{c 2}
$$

and

$$
\widetilde{r}_{0} \cos \widetilde{\alpha}_{0}=k r_{0} \cos \alpha_{0}-(k-1) r_{c 2} \cos \alpha_{c 2} .
$$

So, from (36) to (38), one has

$$
(k-1)\left(r_{c 1} \sin \alpha_{c 1}-r_{c 2} \sin \alpha_{c 2}\right)=0
$$

and

$$
(k-1)\left(r_{c 1} \cos \alpha_{c 1}-r_{c 2} \cos \alpha_{c 2}\right)=0
$$

which leads to $k \equiv 1$ unless $r_{c 1}=r_{c 2}$ and $\alpha_{c 1}=\alpha_{c 2}$. So for a multislit system, as long as there exist two slits that have different $r_{c i}$ or $\alpha_{c i}$, the uniqueness of calibration is guaranteed. The above derivations in multislit case are similar to that in [13] except that: 1) in this work, we discuss a simplified 2-D acquisition geometry and 2) the rotational component is the point object, rather than the camera-detectors in [13].

\section{NUMERICAL VALIDATION}

We validate the method described in Section II-B by applying it to the calibration of the slit-slat SPECT system. The numerical validation consists of following steps.

1) Derive the analytical expression of $J$ according to its definition in (12) and the geometrical projection model defined in (20).

2) Choose the number of projections. The projections are always evenly spaced over $360^{\circ}$ range.

3) Numerically define certain parameter set $\boldsymbol{\Gamma}$ and calculate corresponding $J(\boldsymbol{\Gamma})$.

4) Perform SVD for $J$ and obtain $U, S$, and $V$.

5) Calculate the STD of each parameter from (18).

\section{A. Uniqueness Analysis}

For a system that has only one slit and only one point source is used, the parameter set is $\boldsymbol{\Gamma}=\left(r_{s}, \alpha_{s}, r_{0}, \alpha_{0}, r_{c 1}, \alpha_{c 1}\right)$. Since some of the parameters may change over different experiments and the Jacobian matrix $J$ varies with $\Gamma$, numerical evaluations were performed by uniformly sampling 1000 realizations of $\boldsymbol{\Gamma}$ within certain ranges to avoid picking an exception case. Firstly, the ideal values of the parameters were taken from the design of the slit-slat SPECT system. Secondly, the ranges of the parameters were defined according to the observation in actual study and summarized in Table I.

For each sampled $\Gamma, 60$ projections $p_{i}(\Gamma)$ were used. $J(\Gamma)$ and its SVD components $U, S$, and $V$ were calculated using Matlab (Mathworks, Natick, MA). In case of one slit, the mean and STD of the singular values calculated from 1000 realizations were $\{607.7422 \pm 21.4052,72.2318 \pm 17.7968$, 
TABLE I

CALIBRation PARAMETERS' IDEAL VALUES AND RANGES USED IN Numerical EVAluations For a CALIBRATION SETUP With ONE POINT SOURCE AND ONE SLIT. The IDEAL VALUES WERE TAKEN From SySTEM DESIGN, AND THE RANGES WERE CHOSEN FROM THE OBSERVATION IN ACTUAL STUdy. THERE WAS No LiMIT ON $\alpha_{0}$

\begin{tabular}{ccc|ccc}
\hline parameter & $\begin{array}{c}\text { ideal value } \\
(\mathrm{mm})\end{array}$ & $\begin{array}{c}\text { range } \\
(\mathrm{mm})\end{array}$ & parameter & $\begin{array}{c}\text { ideal value } \\
\left({ }^{\circ}\right)\end{array}$ & $\begin{array}{c}\text { range } \\
\left({ }^{\circ}\right)\end{array}$ \\
\hline$r_{s}$ & 8 & $(6,10)$ & $\alpha_{s}$ & 10 & $(-15,15)$ \\
$r_{0}$ & 0 & $(0,2)$ & $\alpha_{0}$ & arbitrary & $(0,360)$ \\
$r_{c 1}$ & 30 & $(25,35)$ & $\alpha_{c 1}$ & 0 & $(-10,10)$ \\
\hline
\end{tabular}

$10.8685 \pm 2.4451,0.2151 \pm 0.0891,0.1609 \pm 0.0745$, $0.0000 \pm 0.0000\}$. The minimums of the singular values were $\{567.9884,38.9262,6.9582,0.0780,0.0274,0.0000\}$. It was observed that the first 5 singular values were always nonzero, whereas the smallest one $\sigma_{6}$ was always zero, which implies the singularity of $J$ and thus the nonuniqueness of the calibration problem. The right singular vector that corresponds to $\sigma_{6}$ is $\mathbf{v}_{6}$. The calculated mean and STD of $\left|\mathbf{v}_{6}\right|$ over the 1,000 realizations $(|\cdot|$ denotes taking the absolute value for each element) were $\{0.2977 \pm 0.0868,0.0000 \pm 0.0000,0.6719 \pm 0.2845$, $0.5377 \pm 0.2873,0.0000 \pm 0.0000,0.0000 \pm 0.0000\}$, corresponding to $\left\{r_{s}, \alpha_{s}, r_{0}, \alpha_{0}, r_{c 1}, \alpha_{c 1}\right\}$ respectively. The minimal values of $\left|\mathbf{v}_{6}\right|$ elements were $\{0.0474,0.0000,0.0029,0.0006$, $0.0000,0.0000\}$. According to (15), the calibration problem has multiple solutions for $r_{s}, r_{0}$ and $\alpha_{0}$ because they always have nonzero components in $\mathbf{v}_{6}$, whereas $\alpha_{s}, r_{c}$ and $\alpha_{c}$ can be determined uniquely.

When the SPECT system has two slits, $\Gamma$ is extended to $\boldsymbol{\Gamma}=\left(r_{s}, \alpha_{s}, r_{0}, \alpha_{0}, r_{c 1}, \alpha_{c 1}, r_{c 2}, \alpha_{c 2}\right)$. The two slits were chosen to be in the opposing side. Sixty projections were defined per each slit, 1000 realizations of $\Gamma$ were sampled, and corresponding $J, U, S, V$ were calculated. The mean and STD of the singular values were $\{623.5560 \pm 17.2241,591.7222 \pm 10.4969$, $102.5962 \pm 21.4233,14.2239 \pm 2.1431,6.1013 \pm 1.8625$, $1.5842 \pm 0.5194,0.2200 \pm 0.0758,0.1428 \pm 0.0547\}$, calculated from 1000 realizations. The minimums of the singular values were $\{591.6739,569.3036,58.7474,9.8710,2.6905$, $0.2082,0.0866,0.0169\}$. No zero singular value was observed in any of the 1000 realizations, so we conclude the uniqueness condition is satisfied for all the cases evaluated. The numerical study results of the uniqueness for both one-slit and two-slit cases coincide with that from analytical study in Section III-C.

\section{B. Accuracy Analysis}

Although the uniqueness condition is satisfied for a two-slit system with one point source, as shown in the last paragraph in Section IV-A, the accuracy of the estimated parameters may not be good enough because several small singular values appear in $S$. As a demonstration, Table II shows the singular values and right singular vectors calculated from one realization. From Table II one can intuitively predict the accuracy of each parameter. For example, looking at the $v_{1}$ and $v_{2}$ columns and $\alpha_{c 1}$ and $\alpha_{c 2}$ rows, we see that the right singular vectors that correspond to the largest two singular values (608.27 and 591.40) are only related to $\alpha_{c 2}$ and $\alpha_{c 1}$ respectively (both have weight $=0.99$ ), so the estimations of these two parameters are expected to be most accurate, whereas the $v_{7}$ and $v_{8}$ columns and $r_{c 1}$ and $r_{c 2}$ rows show that the smallest two singular values (0.10 and 0.07) have considerable weights on $r_{c 1}$ (weight $=-0.69$ and -0.44 ) and $r_{c 2}$ (weight $=-0.68$ and 0.44 ), which implies the estimation of $r_{c 1}$ and $r_{c 2}$ are less accurate. Similarly, the estimations of $r_{0}$ and $\alpha_{0}$ are also less accurate. The last column of Table II shows the calculated STD from (18), indicating the estimation uncertainty for each parameter.

\section{Calibration Optimization}

Calibration accuracy depends on several factors, including the number of projections per each point source, which is denoted as $\mathbb{N}$, the number of point sources used, the start-positions of the point sources, and any a priori knowledge of system geometry that may be useful to improve the accuracy. It is important to find a simple and reliable setup that enables optimal calibration accuracy. Here, we use the proposed method to guide the search.

The prototype SPECT system we developed had 8 slits, which were evenly spaced over $360^{\circ}$ in the trans-axial direction by design. When one point source was used in the calibration, the ideal values of $r_{s}, \alpha_{s}, r_{0}, \alpha_{0}$, and $r_{c i}$ were still the same as shown in Table $\mathrm{I}$, where $\mathrm{i}=1, \ldots, 8$, except that the ideal value of $\alpha_{c i}$ was $i \times 45^{\circ}$, and the range of $\alpha_{c i}$ was $\left(i \times 45^{\circ}-10^{\circ}, i \times\right.$ $45^{\circ}+10^{\circ}$ ).

The impact of projection number $\mathbb{N}$ was investigated first. $\mathbb{N}=15,30,60,120,240,360,480,1200,2400$, and 3600 were chosen. For each $\mathbb{N}, 1000$ realizations of $\Gamma=\left(r_{s}, \alpha_{s}, r_{0}, \alpha_{0}, r_{c 1}, \alpha_{c 1}, \ldots, r_{c 8}, \alpha_{c 8}\right)$ were sampled which were uniformly distributed over the defined ranges; and the corresponding $J, U, S$ and $V$ were calculated. The STD of all the parameters were calculated based on (18). STD as a function of $\mathbb{N}$ is plotted in Fig. 3. Interestingly, all the plots show linear relationships on a log-log scale. The plot corresponding to $\alpha_{0}$ has relatively larger fluctuations, which may be because when $r_{0}$ is very close to 0 , the estimation of $\alpha_{0}$ is very unstable. From Fig. 3, one can quantitatively predict the achievable calibration accuracy with a given $\mathbb{N}$.

In addition to increasing $\mathbb{N}$, there may exist other ways to improve the calibration accuracy. For example, adding additional point source(s) increases the number of measured projections with same $\mathbb{N}$; Or measuring $r_{s}$ first and using it as a priori knowledge in the calibration; Or acquiring two groups of projections with the same $r_{s}$ and $\alpha_{s}$, and known relative position which can be used as a priori condition.

Six cases of different calibration setups were investigated to search for the optimal calibration protocol, which are as follows.

- Case 1: one point source, no other additional conditions.

- Case 2: one point source, with a priori ROR.

- Case 3: two point sources on different orbits, with the same $r_{s}$ and $\alpha_{s}$. The relative position between CORs were known. The ideal positions of the two CORs were $(-10 \mathrm{~mm}, 0)$ and $(10 \mathrm{~mm}, 0)$, respectively.

- Case 4: two point sources with the same $r_{s}$ and $\alpha_{s} . r_{s}$ and the relative position between CORs were known. The ideal CORs were at $(-10 \mathrm{~mm}, 0)$ and $(10 \mathrm{~mm}, 0)$, respectively.

- Case 5: three point sources with the same $r_{s}$ and $\alpha_{s} . r_{s}$ and the relative position between CORs were known. The 
TABLE II

Singular Vectors $\left(v_{1}, \ldots, v_{8}\right)$ (Row 2 To Row 9) AND Singular VAlues $s_{i}$ (LASt Row) FOR the CALibration With 1 Point Source, 2 Slits AND

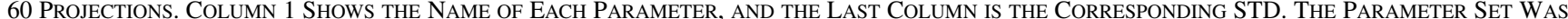
$\boldsymbol{\Gamma}=\left(r_{s}, \alpha_{s}, r_{0}, \alpha_{0}, r_{c 1}, \alpha_{c 1}, r_{c 2}, \alpha_{c 2}\right)=\left(6.4804 \mathrm{MM},-10.4310^{\circ}, 0.9875 \mathrm{MM},-143.0286^{\circ}, 32.5771 \mathrm{MM},-7.9037^{\circ}, 34.1245 \mathrm{MM}, 172.9058^{\circ}\right)$

\begin{tabular}{crrrrrrrrr}
\hline parameter & $v_{1}$ & $v_{2}$ & $v_{3}$ & $v_{4}$ & $v_{5}$ & $v_{6}$ & $v_{7}$ & $v_{8}$ & \multicolumn{1}{c}{ STD } \\
\hline$r_{s}$ & 0.00 & 0.00 & 0.00 & 0.97 & 0.00 & 0.00 & -0.23 & 0.00 & $0.45 \mathrm{~mm}$ \\
$\alpha_{s}$ & 0.01 & -0.01 & 0.99 & 0.00 & 0.00 & 0.00 & 0.00 & 0.00 & $0.16^{\circ}$ \\
$r_{0}$ & 0.00 & 0.00 & 0.00 & 0.00 & -0.48 & -0.68 & 0.00 & 0.55 & $1.65 \mathrm{~mm}$ \\
$\alpha_{0}$ & 0.00 & 0.00 & 0.00 & 0.00 & 0.41 & -0.73 & 0.00 & -0.55 & $95.22^{\circ}$ \\
$r_{c 1}$ & 0.00 & 0.00 & 0.00 & -0.17 & -0.55 & 0.00 & -0.69 & -0.44 & $1.86 \mathrm{~mm}$ \\
$\alpha_{c 1}$ & 0.00 & 0.99 & 0.01 & 0.00 & 0.00 & 0.02 & 0.00 & 0.00 & $0.24^{\circ}$ \\
$r_{c 2}$ & 0.00 & 0.00 & 0.00 & -0.16 & 0.56 & 0.02 & -0.68 & 0.44 & $1.86 \mathrm{~mm}$ \\
$\alpha_{c 2}$ & 0.99 & 0.00 & 0.01 & 0.00 & 0.00 & -0.02 & 0.00 & 0.00 & $0.23^{\circ}$ \\
\hline$s_{i}$ & 608.27 & 591.40 & 65.96 & 10.70 & 3.22 & 0.86 & 0.10 & 0.07 & \\
\hline
\end{tabular}

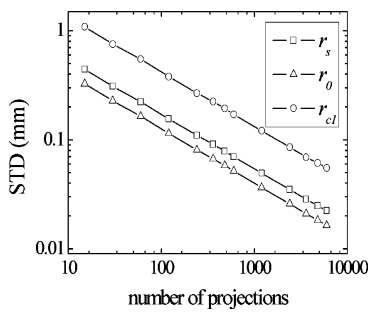

(a)

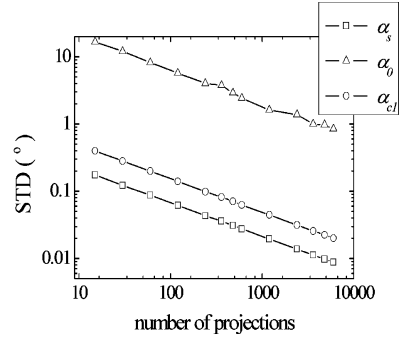

(b)
Fig. 3. STD of (a) the length values, $r_{s}, r_{0}$ and $r_{c 1}$ and (b) the angle values, $\alpha_{s}, \alpha_{0}$ and $\alpha_{c 1}$, as a function of number of projections $(\mathbb{N})$. Plots of $r_{c i}$ and $\alpha_{c i}, \mathrm{i}=2, \ldots, 8$ are very similar to those of $r_{c 1}$ and $\alpha_{c 1}$ respectively and thus are not shown in the figure. The log-log scale was used for all the plots.

CORs were arranged in a straight line, and the ideal values were at $(-10 \mathrm{~mm}, 0),(0,0)$ and $(10 \mathrm{~mm}, 0)$, respectively.

- Case 6: three point sources which the same $r_{s}$ and $\alpha_{s} . r_{s}$ and the relative position between CORs are known. The ideal CORs were at $(-5 \mathrm{~mm},-8.67 \mathrm{~mm}),(-5 \mathrm{~mm}, 8.67$ $\mathrm{mm})$ and $(10 \mathrm{~mm}, 0)$, respectively, which form an equilateral triangle.

For each of the above cases, we followed the similar procedure as that used to obtain Fig. 3. $\mathbb{N}=60,120,360,600,1200$, and 3600 were chosen. STD of calibration parameters as a function of $\mathbb{N}$ are shown in Fig. 4. Plots of $r_{s}$ and $\alpha_{s}$ are not shown because the accuracy of these two parameters has no impact to the system performance. Rather than showing the plots for $\operatorname{STD}\left(r_{c i}\right)$ and $\operatorname{STD}\left(\alpha_{c i}\right)$ of each slit one by one, Fig. 4(c) and (d) show the maximum values over the 8 slits.

All the curves shown in Fig. 4 were fitted to the following equation:

$$
\mathrm{STD}=\frac{A}{\sqrt{\mathbb{N}}}
$$

where $A$ is a fitting parameter. The goodness-of-fit was evaluated by the coefficient of determination $R^{2}$. For all the cases, we have $R^{2}>0.998$ for $r_{0}, r_{c}$ and $\alpha_{c}$ and $R^{2}>0.97$ for $\alpha_{0}$, which empirically verified all the linear curves in the log-log scaled plots shown in both Figs. 3 and 4.

The relationship between STD and $\mathbb{N}$ in (41) can be explained by a theorem in nonlinear regression theory, which was proposed by Gallant in [21]. With the notations defined in this paper, the theorem is expressed as follows.

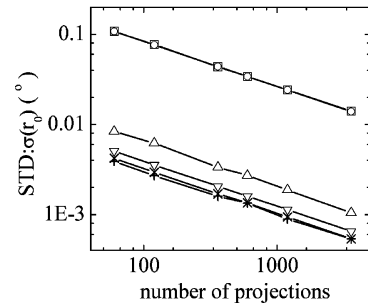

(a)

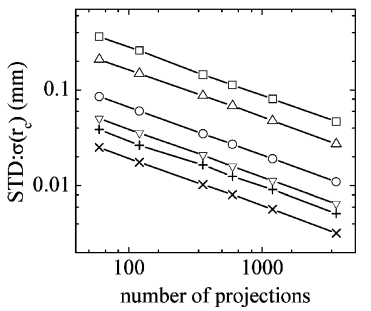

(c)

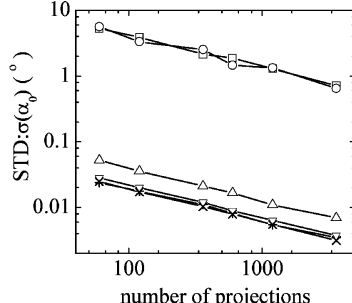

(b)

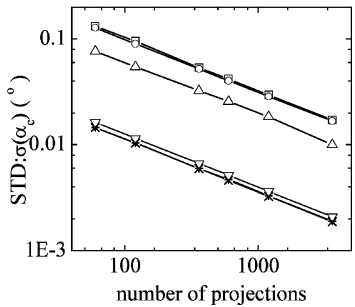

(d)

$$
\begin{array}{ll}
-\square-\text { case } 1 & -\nabla-\text { case } 4 \\
-\circ-\text { case } 2 & -+- \text { case } 5 \\
-\triangle-\text { case } 3 & -\times- \text { case } 6
\end{array}
$$

Fig. 4. STD of (a) $r_{0}$, (b) $\alpha_{0}$, (c) $r_{c}$, and (d) $\alpha_{c}$ are shown as a function of number of projections $\mathbb{N}$ for calibration setup cases 1 to 6 .

Theorem 1: the estimation $\Gamma^{\dagger}$ converges almost surely to the true value $\Gamma^{*}$ and may be characterized as

$$
\Gamma^{\dagger}=\Gamma^{*}+\left(J^{\mathrm{T}} J\right)^{-1} J^{\mathrm{T}} \mathbf{e}+\boldsymbol{\xi}_{\mathbb{N}}
$$

where $\sqrt{\mathbb{N}} \boldsymbol{\xi}_{\mathbb{N}}$ converges in probability to zero. It should be pointed out that this theorem requires that: 1) $e_{i}$ are independent and normally distributed with zero mean and variance $\sigma_{i}^{2}$, as is assumed in this study; and 2) $f_{i}(\Gamma)$ satisfy certain regular conditions [21]. A full proof of such regular conditions is not included in this paper.

Table III shows the minimum required total number of projections $\mathbb{N}_{P}$, which is the product of $\mathbb{N}$ and the number of point sources, for achieving a desired calibration accuracy. $\mathbb{N}_{P}$ can be used as an absolute index to judge the goodness of the calibration configuration. The high $\mathbb{N}_{P}$ values for case 1 and 2 show that using one point source can hardly provide accurate calibration, although the uniqueness condition are satisfied. The $\sigma_{r_{c}}$ column shows a priori knowledge of $r_{s}$ (case $\left.4,5,6\right)$ greatly improved the estimation accuracy of $r_{c}\left(\mathbb{N}_{P}\right.$ dropped from 7317 to 
TABLE III

Minimum Number of Projections $\mathbb{N}_{P}$ Required to Achieve a Desired Calibration Accuracy for the 6 Calibration Setup Cases. $\mathbb{N}_{P}$ IS THE PRODUCT OF $\mathbb{N}$ AND NUMBER OF POINT SOURCES

\begin{tabular}{cccccc}
\hline case index & number of point sources & $\sigma_{r_{0}}<0.017 \mathrm{~mm}$ & $\sigma_{\alpha_{0}}<0.054^{\circ}$ & $\sigma_{r_{c}}<0.033 \mathrm{~mm}$ & $\sigma_{\alpha_{c}}<0.095^{\circ}$ \\
\hline 1 & 1 & 2470 & 127151 & 7317 & 267 \\
2 & 1 & 2394 & 53954 & 400 & 249 \\
3 & 2 & 29 & 104 & 4908 & 418 \\
4 & 2 & 9 & 39 & 268 & 22 \\
5 & 3 & 9 & 35 & 183 & 12 \\
6 & 3 & 11 & 39 & 100 & 14 \\
\hline
\end{tabular}

400 with one point source and from 4908 to 268 with two point sources). Overall, case 4,5 , and 6 provide satisfactory accuracy for all the parameters with small $\mathbb{N}$ value. Since corresponding total number of projections $\mathbb{N}_{P}=268,183$ and 100, the minimum required projection per point source is $\mathbb{N}=134,92$, and 34 respectively.

\section{EXPERIMENTAL VALIDATION}

Several experiments were performed to evaluate the proposed calibration methods. Firstly, by taking the advantage of the existing PET system, ROR and COR were determined accurately via PET point source measurements and served as a priori condition (ROR) and standard to judge the calibration accuracy (COR), respectively. Secondly, SPECT point source projections with different calibration setups were acquired and geometrical calibrations were performed. The calibration results of each setup were compared in terms of accuracy. Finally, an experimental phantom study was conducted to test the calibration accuracy with reconstructed images.

\section{A. A Priori Information From PET Imaging}

A ${ }^{68}$ Ge point source $(\phi 1 \mathrm{~mm}, \mathrm{~L} 1 \mathrm{~mm}$, Isotope Products Laboratory, Burbank, CA) was mounted on the linear stage and moved $20 \mathrm{~mm}$ in both trans-axial and axial directions with $5 \mathrm{~mm}$ step interval, and a PET image was acquired in each step. Each point source image was fitted to a 2-D Gaussian function, and the center of the point source in the image was determined. The PET imaging accuracy was tested by calculating the distances between the centers and comparing them with the moving distance of the stage.

The point source was afterwards rotated by a rotation stage for 12 steps at $30^{\circ}$ step. By measuring the centroids of the PET images at each step and fitting them into a round curve, the ROR and COR were determined.

With point source moving $5 \mathrm{~mm}$ in each step in trans-axial direction (from bottom to top since the current installed stage only allowed vertical movement), the measured distance from FBP reconstructed PET images was $4.986 \pm 0.023 \mathrm{~mm}$. In the axial direction, the measured value was $4.983 \pm 0.035 \mathrm{~mm}$. Therefore, the distance measured in PET images has acceptable accuracy $(<0.05 \mathrm{~mm})$ for our studies. The fitted ROR and COR using PET images from point-source-rotation studies were $7.626 \mathrm{~mm}$ and $(-0.420 \mathrm{~mm},-0.227 \mathrm{~mm})$ respectively, which were used as reference values in the following calibration studies.
TABLE IV

ESTIMATION ERROR (IN MM) OF THE CALIBRATED COR VALUES WITH DifFERENT $\mathbb{N}$ IN CONFIGURATION CASES 1 THROUGH 5. DATA WERE Compared to the True COR VAlue, $(-0.420 \mathrm{~mm},-0.227 \mathrm{~mm})$ MEASURED FROM PET IMAGING

\begin{tabular}{cccccc}
\hline $\mathbb{N}$ & case 1 & case 2 & case 3 & case 4 & case 5 \\
\hline 30 & 0.222 & 0.254 & 0.275 & 0.169 & 0.078 \\
60 & 0.262 & 0.276 & 0.295 & 0.091 & 0.057 \\
120 & 0.262 & 0.249 & 0.246 & 0.076 & 0.033 \\
\hline
\end{tabular}

\section{B. SPECT Calibrations}

A $5 \mathrm{mCi}{ }^{57}$ Co point source ( $\phi 1 \mathrm{~mm}$, L $1 \mathrm{~mm}$, Isotope Products Laboratory, Burbank, CA) was used to acquire the SPECT data for calibration. By mounting the point source onto the same rotation stage, three groups of SPECT data were acquired, with the original COR position (orbit 1), moving the COR $10 \mathrm{~mm}$ above (orbit 2) and $10 \mathrm{~mm}$ below (orbit 3 ) relative to the original position. For each orbit, 120 projections were acquired with $3^{\circ} / \mathrm{step}$ with $10 \mathrm{~s}$ acquisition time per projection.

To compare the results with the numerical studies in Section IV-B, five calibration configurations were defined as listed below. These 5 configurations matched case 1 to 5 in Section IV-B. Case 6 in Section IV-B was not tested because the current experimental setup only supported point source's translational movement in one direction.

- Case 1: use orbit 2, ROR is unknown.

- Case 2: use orbit 2, ROR is known.

- Case 3: use orbit 1 and 3, ROR is unknown.

- Case 4: use orbit 1 and 3, ROR is known.

- Case 5: use orbit 1,2 and 3, ROR is known.

For each of the 5 cases, 3 different $\mathbb{N}(30,60$, and 120) per source location were investigated, and the projections were always evenly spaced over $360^{\circ}$ span. The calibration was thus carried out 15 times with the combination of each case and each $\mathbb{N}$. The minimization of (2) was performed in Matlab using the Levenberg-Marquardt algorithm [22]. In each calibration, the geometrical distance between the calibrated COR and true value was calculated and listed in Table IV, as an absolute accuracy measurement for COR estimation. Since the SAPs could not be measured similarly in PET imaging, the measured values with 120 projections per source location in case 5 were taken as the reference values since they were considered the most accurate ones. For each of the other 14 situations, the distances between the estimated SAPs and the true values were calculated for all the 8 slits, and the average and the standard deviation of those 8 distance values are listed in Table V. By comparing the achievable accuracy of different calibration setup cases, the experimental measurements show similar trend to the mathematical 
TABLE V

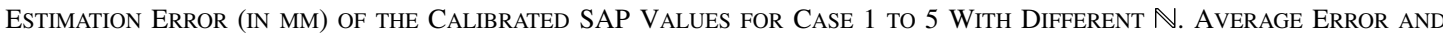
STD for 8 Slits are Shown. SAP Values From Case 5 AND With 120 Projections Were Used as the True Values

\begin{tabular}{cccccc}
\hline $\mathbb{N}$ & case 1 & case 2 & case 3 & case 4 & case 5 \\
\hline 30 & $0.738 \pm 0.368$ & $0.692 \pm 0.258$ & $0.658 \pm 0.348$ & $0.096 \pm 0.038$ & $0.032 \pm 0.019$ \\
60 & $0.770 \pm 0.375$ & $0.697 \pm 0.261$ & $0.657 \pm 0.368$ & $0.097 \pm 0.046$ & $0.016 \pm 0.007$ \\
120 & $0.758 \pm 0.411$ & $0.690 \pm 0.258$ & $0.637 \pm 0.363$ & $0.093 \pm 0.041$ & N/A \\
\hline
\end{tabular}

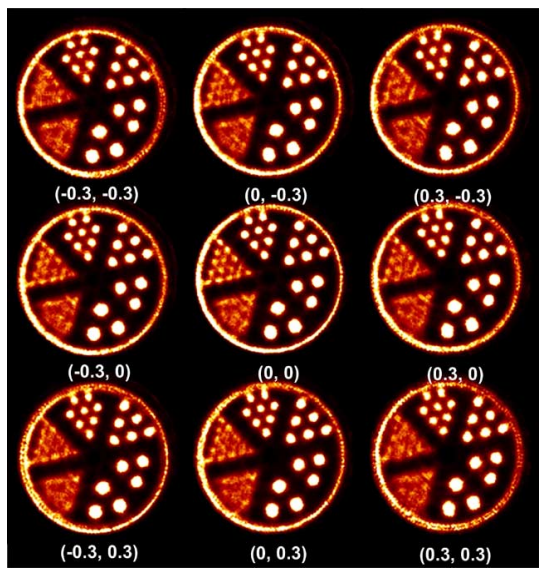

Fig. 5. Reconstructed ultra-micro hot-rod phantom images with 30 iterations and 8 subsets with different COR values. The central one was reconstructed with the properly calibrated COR value. The values shown below each image indicates the displacement error that was manually add to the COR value, in $x$ and $y$ direction. For fair comparison, same absolute scaling values was used to display all the images.

predictions as shown in Fig. 4 and Table III. With known relative point source's location and ROR as the a priori conditions and 120 projections per each point source location, the use of at least two groups of point source measurements can provide satisfactory calibration accuracy $(\sim 0.10 \mathrm{~mm}$ for both COR and SAPs).

\section{Phantom Experiment}

An ultra-micro hot-rod phantom (Data Spectrum, Inc., Hillsborough, NC) was used to demonstrate the effects of calibration. The diameters of the hot rods were 2.4, 2.0, 1.7. 1.35, 1.0, and $0.7 \mathrm{~mm}$, respectively, from the largest to the smallest. The phantom was filled with $5.5 \mathrm{mCi} 99 \mathrm{mTc}$ solution. It was rotated for 120 steps at $3 \%$ step, and scanned for $40 \mathrm{~s}$ at each step. SPECT data were acquired in the $120-160 \mathrm{keV}$ energy window. Images were reconstructed with an iterative ordered subsets expectation maximization algorithm [23] and system matrix derived from Monte Carlo simulation [24]. The calibrated slit collimator geometry was modeled in the PSF system matrix, and the data were firstly reconstructed with the correctly calibrated COR value, and then with several "wrong" COR values which had estimation errors that were artificially introduced with 0.3 $\mathrm{mm}$ and/or $-0.3 \mathrm{~mm}$ in $x$ and/or $y$ direction. The reconstructed images are shown in Fig. 5. The $1.0 \mathrm{~mm}$ hot rod pattern is clearly visualized with proper calibrated COR values, and $0.3 \mathrm{~mm}$ error in COR estimation causes visible image resolution loss to the studied SPECT system.

\section{DISCUSSION}

With the method proposed in this study, we have shown that one rotating point source is insufficient to calibrate a slit-slat SPECT system with only one slit. This conclusion agrees with that obtained from analytical derivation. In case of multiple slits, although the uniqueness condition can be satisfied, the proposed method reveals that estimation of some calibration parameters are inaccurate with one point source. Using more than one point sources and with the a priori knowledge of ROR and relative positions between point sources, calibration accuracy can be substantially improved. The proposed method has been used to guide the optimization of calibration with different calibration source configurations. The advantages of the proposed method include 1) the uniqueness and accuracy characteristics of the calibration can be assessed in a unified framework, which are respectively related to the zero and small singular values of the Jacobian matrix of the cost function; 2) the SVD components $S$ and $V$ of the Jacobian matrix provide a straightforward method to estimate the accuracy of each individual calibration parameter; and 3) the proposed method is flexible and can be adapted to any type of calibration source arrangement or calibration phantom design, as long as the geometrical projection model can be derived and the least square cost function is differentiable, so that the Jacobian matrix can be calculated. This is especially useful for handling nonstandard acquisition geometry, in which the analytical approach may encounter difficulties.

In terms of deriving the uniqueness condition of the calibration, analytical method can provide a general conclusion when the calibration parameter set $\Gamma$ satisfies certain conditions. In comparison, conclusion from SVD evaluation is only valid at the evaluated expansion point $\Gamma^{\dagger}$ as defined in (6) and (7), so one has to perform the evaluation multiple times by using a different $\Gamma^{\dagger}$ each time over a certain range to reach a general conclusion. However, in practice most of the nonlinear least square fitting algorithms (e.g., the Levenberg-Marquardt algorithm) are implemented in an iterative way, and at each iterative step a linear least square fitting is performed, the proposed method can be incorporated into the iteration procedure and predict the behavior of the fitting algorithm at each step.

It needs to be noted that in (9) we choose the estimation solution $\Gamma^{\dagger}$ as the expansion point, rather than the true solution $\Gamma^{*}$ for the following reasons: 1) the Jacobian matrix $J$ has to be evaluated at the expansion point, and in practice both the true solution and $J$ are unknown if the expansion is performed near $\left.\Gamma^{*} ; 2\right)$ the nonlinear least square fitting algorithms also requires performing Taylor expansion near the estimated solution in the last iterative step; and 3) when $\Gamma^{\dagger}$ is close to $\Gamma^{*},\left.J\right|_{\Gamma=\Gamma^{\dagger}}$ is approximately equal to $\left.J\right|_{\Gamma=\Gamma^{*}}$ to the first order of approximation, 
so numerically there is no significant difference between performing expansion near $\boldsymbol{\Gamma}^{\dagger}$ and near $\boldsymbol{\Gamma}^{*}$.

In Fig. 5, we have shown that a $0.3 \mathrm{~mm}$ estimation error of COR can produce clearly visible resolution loss in the reconstructed image on the system we studied. It was also reported in literatures that the estimation error of different individual parameters may affect different aspects of the reconstruction image. For example, Gullberg et al. observed in [7] that the focus-to-center distance in cone beam tomography, which is equivalent to $r_{c}$ in this study, is more difficult to be accurately calibrated than the COR. Although the accuracy of $r_{c}$ estimation does not affect the image resolution as much as COR does, the reconstructed image may be magnified or minified by the estimation error of $r_{c}$. Bequé et al. also demonstrated in [12] that in pinhole SPECT imaging, the correlated error of the electrical shift in the axial direction and the detector's tilting angle produces large distortions of the reconstructed image. They also showed that the correlated error of the mechanical offset and the electrical shift in the trans-axial direction causes less image distortion, but results in a rotation of the reconstructed image around the axis of rotation. Apparently, neither the distortion nor the rotation of the reconstructed image are desired. Knowing that most of the imaging systems are designed to have a stable geometrical structure, the endeavor to carry out the most accurate calibration possible for every parameter is usually justified by the relative long-term benefit of the improved image quality. Nevertheless, a quantitative measure of an inaccurate parameter's impact on the reconstructed image quality would be highly desirable for selecting the most effective and efficient calibration protocol. A quantitative analysis of the error propagation from calibration to reconstructed images will be studied in our future work.

The proposed method only investigates the impact of random error in the measured data on the calibration accuracy. The impact of systematic error that may exist for the predetermined parameters, e.g., the imaging system's geometry information in the source projection (20) can't be estimated with the proposed method. For example, an averaged DOI value was used in (20) to calculate $R$ for all the crystals, which may be inaccurate for oblique incident photons, especially for those crystals near the edge of a detector block. Reduction of systematic error can be done separately. For example, using crystal and point source location dependent DOI values that can be either derived from Monte-Carlo simulation or from experimental measurement may further improve the calibration accuracy.

\section{CONCLUSION}

We have presented a new method to quantitatively investigate the uniqueness and accuracy of SPECT geometrical calibration. The method is based on SVD analysis of the Jacobian matrix of a least square calibration cost function. With this method, the uniqueness of calibration can be identified by assessing the nonsingularity of the Jacobian matrix, the uncertainty of the calibration parameters can be quantified through analyzing the SVD components, and a calibration configuration can be evaluated by a chosen figure-of-merit, e.g., the minimum required number of projection samplings for a given desired calibration accuracy.
The proposed method was applied to a slit-slat SPECT system and was validated through analytical studies, numerical evaluations and phantom experiments. Since there is no special constraint on data collection mechanism or collimation geometry, this method is expected to be applicable to general SPECT and CT systems for calibration accuracy assessment and calibration protocol optimization.

\section{APPENDIX}

PROOF OF (23)-(28)

This appendix derives (23)-(28) by using (21) and (22). Substituting (22) into (21) and rearranging yields

$$
\begin{aligned}
& A_{1} \cos \left(\alpha_{s}+\widetilde{\alpha}_{s}+2 \theta\right)+A_{2} \sin \left(\alpha_{s}+\widetilde{\alpha}_{s}+2 \theta\right) \\
& \quad+B_{1} \cos \left(\alpha_{s}+\theta\right)+B_{2} \sin \left(\alpha_{s}+\theta\right)+B_{3} \cos \left(\widetilde{\alpha}_{s}+\theta\right) \\
& \quad+B_{4} \sin \left(\widetilde{\alpha}_{s}+\theta\right)+C=0
\end{aligned}
$$

where

$$
\begin{aligned}
A_{1}= & r_{s} \widetilde{r}_{s}\left(\widetilde{r}_{c} \cos \widetilde{\alpha}_{c}-r_{c} \cos \alpha_{c}\right) \\
A_{2}= & r_{s} \widetilde{r}_{s}\left(\widetilde{r}_{c} \sin \widetilde{\alpha}_{c}-r_{c} \sin \alpha_{c}\right) \\
B_{1}= & r_{s}\left(r_{c} \sin \alpha_{c}-R\right)\left(\widetilde{r}_{0} \sin \widetilde{\alpha}_{0}-\widetilde{r}_{c} \sin \widetilde{\alpha}_{c}\right) \\
B_{2}= & r_{s}\left(\widetilde{r}_{0} \widetilde{r}_{c} \sin \widetilde{\alpha}_{0} \cos \widetilde{\alpha}_{c}-\widetilde{r}_{0} \widetilde{r}_{c} \cos \widetilde{\alpha}_{0} \sin \widetilde{\alpha}_{c}+\widetilde{r}_{0} R \sin \widetilde{\alpha}_{0}\right. \\
& \left.-\widetilde{r}_{c} R \sin \widetilde{\alpha}_{c}-\widetilde{r}_{0} r_{c} \sin \widetilde{\alpha}_{0} \cos \alpha_{c}+r_{c} \widetilde{r}_{c} \cos \alpha_{c} \sin \widetilde{\alpha}_{c}\right)
\end{aligned}
$$

$$
B_{3}=\widetilde{r}_{s}\left(\widetilde{r}_{c} \sin \alpha_{c}-R\right)\left(r_{0} \sin \alpha_{0}-r_{c} \sin \alpha_{c}\right)
$$

$B_{4}=\widetilde{r}_{s}\left(r_{0} r_{c} \cos \alpha_{c} \sin \alpha_{0}-r_{0} r_{c} \cos \alpha_{0} \sin \alpha_{c}+r_{0} R \sin \alpha_{0}\right.$ $\left.-r_{c} R \sin \alpha_{c}-r_{0} \widetilde{r}_{c} \sin \alpha_{0} \cos \widetilde{\alpha}_{c}+\widetilde{r}_{c} r_{c} \cos \widetilde{\alpha}_{c} \sin \alpha_{c}\right)$

$$
\begin{aligned}
C= & \left(\widetilde{r}_{0} \sin \widetilde{\alpha}_{0}-\widetilde{r}_{c} \sin \widetilde{\alpha}_{c}\right) \times\left(r_{0} r_{c} \sin \alpha_{0} \cos \alpha_{c}\right. \\
& \left.-r_{0} r_{c} \cos \alpha_{0} \sin \alpha_{c}+r_{0} R \cos \alpha_{0}-r_{c} R \cos \alpha_{c}\right) \\
& -\left(r_{0} \sin \alpha_{0}-r_{c} \sin \alpha_{c}\right) \times\left(\widetilde{r}_{0} \widetilde{r}_{c} \sin \widetilde{\alpha}_{0} \cos \widetilde{\alpha}_{c}\right. \\
& \left.-\widetilde{r}_{0} \widetilde{r}_{c} \cos \widetilde{\alpha}_{0} \sin \widetilde{\alpha}_{c}+\widetilde{r}_{0} R \cos \widetilde{\alpha}_{0}-\widetilde{r}_{c} R \cos \widetilde{\alpha}_{c}\right) \\
& +r_{s} \widetilde{r}_{s}\left(r_{c} \sin \alpha_{c}+\widetilde{r}_{c} \sin \widetilde{\alpha}_{c}\right) \sin \left(\widetilde{\alpha}_{s}-\alpha_{s}\right) \\
& +r_{s} \widetilde{r}_{s}\left(r_{c} \cos \alpha_{c}-\widetilde{r}_{c} \cos \widetilde{\alpha}_{c}\right) \cos \left(\alpha_{s}-\widetilde{\alpha}_{s}\right) .
\end{aligned}
$$

Through a similar derivation as shown in [12], the fact that equation for any $\theta$, (43) holds requires that the part varying with $2 \theta$, the part varying with $\theta$ and the constant part in the left hand of (43) should each individually be equal to 0 . So, we have

$$
\begin{aligned}
A_{1} \cos \left(\alpha_{s}+\widetilde{\alpha}_{s}+2 \theta\right) & \\
+A_{2} \sin \left(\alpha_{s}+\widetilde{\alpha}_{s}+2 \theta\right) & =0 \\
B_{1} \cos \left(\alpha_{s}+\theta\right)+B_{2} \sin \left(\alpha_{s}+\theta\right) & \\
+B_{3} \cos \left(\widetilde{\alpha}_{s}+\theta\right)+B_{4} \sin \left(\widetilde{\alpha}_{s}+\theta\right) & =0 \\
C & =0 .
\end{aligned}
$$

Rewriting (51) as

$$
\begin{aligned}
& \left(r_{c} \cos \alpha_{c}-\widetilde{r}_{c} \cos \widetilde{\alpha}_{c}\right) \cos \left(\alpha_{s}+\widetilde{\alpha}_{s}+2 \theta\right) \\
& =\left(r_{c} \sin \alpha_{c}-\widetilde{r}_{c} \sin \widetilde{\alpha}_{c}\right) \sin \left(\alpha_{s}+\widetilde{\alpha}_{s}+2 \theta\right)
\end{aligned}
$$

which naturally leads to (23) and (24). 
Substituting (23) and (24) into (52) and (53) yields

$$
\begin{aligned}
& r_{s}\left(\widetilde{r}_{0} \sin \widetilde{\alpha}_{0}-r_{c} \sin \alpha_{c}\right) \cos \left(\alpha_{s}+\theta\right) \\
& \quad-r_{s}\left(\widetilde{r}_{0} \cos \widetilde{\alpha}_{0}-r_{c} \cos \alpha_{c}\right) \sin \left(\alpha_{s}+\theta\right) \\
& =\widetilde{r}_{s}\left(r_{0} \sin \alpha_{0}-r_{c} \sin \alpha_{c}\right) \cos \left(\widetilde{\alpha}_{s}+\theta\right) \\
& \quad+\widetilde{r}_{s}\left(r_{0} \cos \alpha_{0}-r_{c} \cos \alpha_{c}\right) \sin \left(\widetilde{\alpha}_{s}+\theta\right) \\
& \left(r_{0} \cos \alpha_{0}-r_{c} \cos \alpha_{c}\right)\left(\widetilde{r}_{0} \sin \widetilde{\alpha}_{0}-r_{c} \sin \alpha_{c}\right) \\
& =\left(\widetilde{r}_{0} \cos \widetilde{\alpha}_{0}-r_{c} \cos \alpha_{c}\right)\left(r_{0} \sin \alpha_{0}-r_{c} \sin \alpha_{c}\right) .
\end{aligned}
$$

Both the left- and the right-hand side of (55) can be rewritten as a cosine function with a specific amplitude and phase. Setting the amplitude and phase part to be equal to each other, respectively, yields

$$
\begin{aligned}
& \widetilde{\alpha}_{s}-\arctan \left(\frac{r_{0} \cos \alpha_{0}-r_{c} \cos \alpha_{c}}{r_{0} \sin \alpha_{0}-r_{c} \sin \alpha_{c}}\right) \\
& =\alpha_{s}-\arctan \left(\frac{\widetilde{r}_{0} \cos \widetilde{\alpha}_{0}-r_{c} \cos \alpha_{c}}{\widetilde{r}_{0} \sin \widetilde{\alpha}_{0}-r_{c} \sin \alpha_{c}}\right) \\
& r_{s}\left(\widetilde{r}_{0} \sin \widetilde{\alpha}_{0}-r_{c} \sin \alpha_{c}\right) \sqrt{1+\left(\frac{\widetilde{r}_{0} \cos \widetilde{\alpha}_{0}-r_{c} \cos \alpha_{c}}{\widetilde{r}_{0} \sin \widetilde{\alpha}_{0}-r_{c} \sin \alpha_{c}}\right)^{2}} \\
& =\widetilde{r}_{s}\left(r_{0} \sin \alpha_{0}-r_{c} \sin \alpha_{c}\right) \sqrt{1+\left(\frac{r_{0} \cos \alpha_{0}-r_{c} \cos \alpha_{c}}{r_{0} \sin \alpha_{0}-r_{c} \sin \alpha_{c}}\right)^{2}} .
\end{aligned}
$$

Considering (56), we have (26)-(28).

Equation (57) holds when $r_{0} \sin \alpha_{0}-r_{c} \sin \alpha_{c} \neq 0$ and $\widetilde{r}_{0} \sin \widetilde{\alpha}_{0}-r_{c} \sin \alpha_{c} \neq 0$. If any of these two items is equal to zero, from (56) and according to the fact that the COR can not be overlapped to the SAP, one can derive that

$$
\begin{aligned}
& r_{0} \sin \alpha_{0}-r_{c} \sin \alpha_{c}=\widetilde{r}_{0} \sin \widetilde{\alpha}_{0}-r_{c} \sin \alpha_{c}=0, \\
& r_{0} \cos \alpha_{0}-r_{c} \cos \alpha_{c} \neq 0, \text { and } \\
& \widetilde{r}_{0} \cos \widetilde{\alpha}_{0}-r_{c} \cos \alpha_{c} \neq 0 .
\end{aligned}
$$

In this case, rather than using a cosine function, one can rewrite (55) as a sine function, which also lead to (26)-(28).

\section{ACKNOWLEDGMENT}

This work utilized the high-performance computational capabilities of the Linux cluster at the Center for Computational Research, State University of New York at Buffalo. The authors would like to thank S. Chen at John Hopkins University for helpful discussions.

\section{REFERENCES}

[1] S. R. Meikle, P. Kench, M. Kassiou, and R. B. Banati, "Small animal SPECT and its place in the matrix of molecular imaging technologies," Phys. Med. Biol., vol. 50, no. 22, pp. R45-R61, 2005.

[2] , M. A. Kupinski and H. H. Barrett, Eds., Small-Animal SPECT Imaging. New York: Springer, 2005.
[3] B. L. Franc, P. D. Acton, C. Mari, and B. H. Hasegawa, "Small-animal SPECT and SPECT/CT: Important tools for preclinical investigation," J. Nucl. Med., vol. 49, no. 10, pp. 1651-1663, 2008.

[4] N. Schramm, G. Ebel, U. Engeland, T. Schurrat, M. Behe, and T. Behr, "High-resolution SPECT using multipinhole collimation," IEEE Trans. Nucl. Sci., vol. 50, no. 3, pp. 315-320, Jun. 2003.

[5] F. J. Beekman, F. van der Have, B. Vastenhouw, A. J. van der Linden, P. P. van Rijk, J. P. H. Burbach, and M. P. Smidt, "U-SPECT-I: A novel system for submillimeter-resolution tomography with radiolabeled molecules in mice," J. Nucl. Med., vol. 46, no. 7, pp. 1194-1200, 2005.

[6] F. P. DiFilippo, "Design and performance of a multi-pinhole collimation device for small animal imaging with clinical SPECT and SPECT-CT scanners," Phys. Med. Biol., vol. 53, no. 15, pp. 4185-4201, 2008.

[7] G. T. Gullberg, B. M. W. Tsui, C. R. Crawford, J. G. Ballard, and J. T. Hagius, "Estimation of geometrical parameters and collimator evaluation for cone beam tomography," Med. Phys., vol. 17, no. 2, pp. 264-272, 1990

[8] J. Li, R. J. Jaszczak, H. Wang, K. L. Greer, and R. E. Coleman, "Determination of both mechanical and electronic shifts in cone beam SPECT," Phys. Med. Biol., vol. 38, no. 6, pp. 743-754, 1993.

[9] P. Rizo, P. Grangeat, and R. Guillemaud, "Geometric calibration method for multiple-head cone-beam SPECT system," IEEE Trans. Nucl. Sci., vol. 41, no. 6, pp. 2748-2757, Dec. 1994.

[10] H. Wang, M. F. Smith, C. D. Stone, and R. J. Jaszczak, "Astigmatic single photon emission computed tomography imaging with a displaced center of rotation," Med. Phys., vol. 25, no. 8, pp. 1493-1501, 1998.

[11] F. Noo, R. Clackdoyle, C. Mennessier, T. A. White, and T. J. Roney, "Analytic method based on identification of ellipse parameters for scanner calibration in cone-beam tomography," Phys. Med. Biol., vol. 45, no. 11 , pp. $3489-3508,2000$.

[12] D. Beque, J. Nuyts, G. Bormans, P. Suetens, and P. Dupont, "Characterization of pinhole SPECT acquisition geometry," IEEE Trans. Med. Imag., vol. 22, no. 5, pp. 599-612, May 2003.

[13] Y. Wang and B. M. W. Tsui, "Pinhole SPECT with different data acquisition geometries: Usefulness of unified projection operators in homogeneous coordinates," IEEE Trans. Med. Imag., vol. 26, no. 3, pp. 298-308, Mar. 2007.

[14] F. P. DiFilippo, "Geometric characterization of multi-axis multi-pinhole SPECT," Med. Phys., vol. 35, no. 1, pp. 181-194, 2008.

[15] S. Metzler and R. Jaszczak, "Simultaneous multi-head calibration for pinhole SPECT," IEEE Trans. Nucl. Sci., vol. 53, no. 1, pp. 113-120, Feb. 2006.

[16] M. Defrise, C. Vanhove, and J. Nuyts, "Perturbative refinement of the geometric calibration in pinhole SPECT," IEEE Trans. Med. Imag., vol. 27, no. Feb., pp. 204-214, Feb. 2008.

[17] S. Metzler, K. Greer, and R. Jaszczak, "Determination of mechanical and electronic shifts for pinhole SPECT using a single point source," IEEE Trans. Med. Imag., vol. 24, no. 3, pp. 361-370, Mar. 2005.

[18] L. v. Smekal, M. Kachelriess, E. Stepina, and W. A. Kalender, "Geometric misalignment and calibration in cone-beam tomography," Med. Phys., vol. 31, no. 12, pp. 3242-3266, 2004.

[19] Y. Shao, R. Yao, T. Ma, and P. Manchiraju, "Initial studies of PETSPECT dual-tracer imaging," in Conf. Rec. IEEE 2007 Nucl. Sci. Symp. Med. Imag. Conf., 2007, vol. 6, pp. 4198-4204.

[20] R. Yao, T. Ma, and Y. Shao, "Lutetium oxyorthosilicate (LSO) intrinsic activity correction and minimal detectable target activity study for SPECT imaging with a lso-based animal PET scanner," Phys. Med. Biol., vol. 53, no. 16, pp. 4399-4415, 2008.

[21] R. A. Gallant, "Testing a subset of the parameters of a nonlinear regression model," J. Am. Stat. Assoc., vol. 70, no. 352, pp. 927-932, 1975.

[22] W. H. Press, S. A. Teukolsky, W. T. Vetterling, and B. P. Flannery, Numerical Recipes in C: The Art of Scientific Computing, 2nd ed. New York: Cambridge Univ. Press, 1992.

[23] H. Hudson and R. Larkin, "Accelerated image reconstruction using ordered subsets of projection data," IEEE Trans. Med. Imag., vol. 13, no. 4, pp. 601-609, Dec. 1994

[24] R. Yao, T. Ma, and Y. Shao, "Derivation of system matrix from simulation data for an animal SPECT with slit-slat collimator," IEEE Trans. Nucl. Sci., vol. 56, no. 5, pp. 2651-2658, Oct. 2009. 